\title{
Visual field defects in vascular lesions of the lateral geniculate body
}

\author{
Cristian Luco, Arnold Hoppe, Mariana Schweitzer, Ximena Vicuña, Aldo Fantin
}

Department of Neurology, School of Medicine, Universidad Católica de Chile, Santiago

C Luco

A Hoppe

A Fantin

Department of

Neurology, School of Medicine, Universidad de Chile, and NeuroOphthalmology Unit, Instituto de

Neurocirugia,

C Luco

M Schweitzer

$X$ Vicuña

Correspondence to: Católica de Chile, Marcoleta 387, Santiago, Chile

Received 4 October 1990 and in revised form 7 March 1991 .

Accepted 26 March 1991
Santiago, Chile

Dr Luco, Department of Neurology, Universidad

\begin{abstract}
Corresponding retinal nerve fibres begin their path in the eyes and end in a single visual cortical cell. Because of this arrangement, lesions in the anterior visual pathway produce incongruent visual field defects and in the posterior pathway congruent field defects. The lateral geniculate body is on the anterior third of the visual pathway. A lesion of this nucleus produces moderately to completely congruent visual field defects. Five patients with ischaemic lesions of the lateral geniculate body are reported. Two patients had a wedgeshaped homonymous hemianopia, two other cases had congruent superior homonymous quadrantic defects and the fifth a quadruple sector defect. The lateral geniculate body has a dual blood supply from the anterior choroidal artery (branch from internal carotid artery) and from the lateral choroidal artery (branch from the posterior cerebral artery). A schematic diagram has been devised which shows that a knowledge of the visual field disrupted can identify the arterial system involved.

The fibres of the visual pathway that carry information from the retina to the calcarine cortex have a systematic spatial arrangement. Lesions in this pathway produce characteristic visual field defects whose analysis frequently allows localisation of the lesion, especially in the anteroposterior axis. One of the most frequently used features in diagnosis is congruency-that is, the similarity between the visual field defect of one eye and the other. Congruency is maximal in cortical lesions and
\end{abstract}

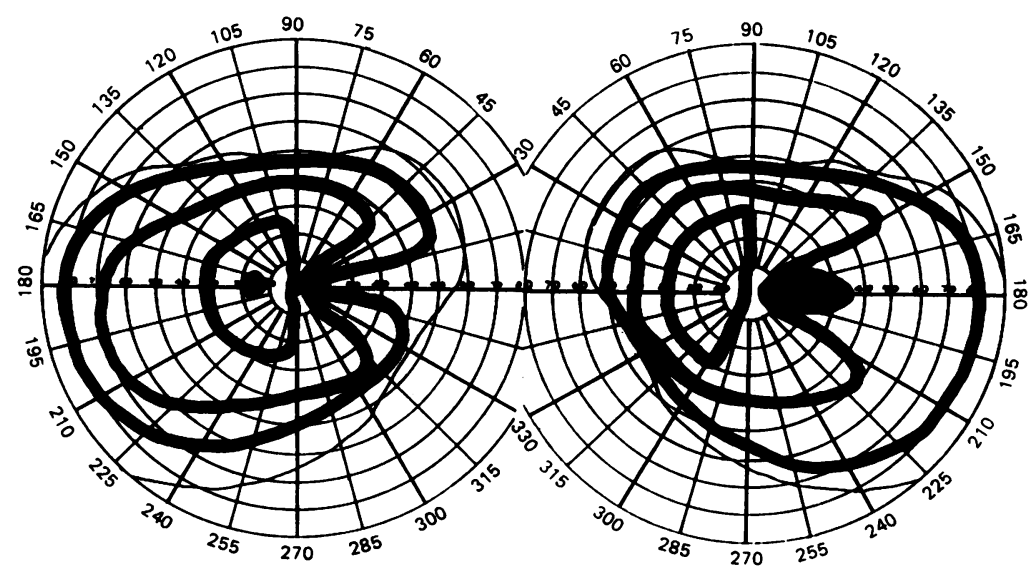

Figure 1 Wedge-shaped, right homonymous hemianopia. Case 1. minimal in optic tract lesions. ${ }^{1}$ A vascular lesion of the lateral geniculate body (LGB) is an exception to the relationship between congruency of the visual field defect and the anteroposterior localisation. Even though the LGB is on the anterior third of the retrochiasmatic visual pathway and a lesion just in front or behind this nucleus produces incongruent field defects, the visual field defect found in these cases is a congruent wedge-shaped homonymous hemianopia or the sparing of a symmetrical wedge-shaped area, and loss of upper and lower quadrants. These visual field defects are infrequent in lesions in other sites of the visual pathway and anatomically almost impossible in a vascular lesion of the visual cortex. Another type of field defect found with LGB lesions is congruent quadrantic contraction of the upper or lower visual field. ${ }^{2}$ Lesions of this nucleus are infrequent and the use of CT enables a more accurate diagnosis.

We report on five patients with vascular lesions of the LGB. Two had the typical wedge-shaped homonymous hemianopia, two other cases had superior congruent homonymous quadrantic defects (quadrantanopsia) and the last one a quadruple sectoranopia. In four cases CT scan revealed a vascular lesion of the LGB and in one case the test was negative.

\section{Case reports}

Case 1

This patient was a 62 year old male with an unremarkable past medical history. In December 1986 he had a severe headache and "visual disturbance". On examination, a few hours later the only abnormality was high blood pressure $(160 / 100 \mathrm{~mm} \mathrm{Hg})$. The neurological examination was normal except for the visual fields. The neurophthalmological examination showed a normal visual acuity, pupillary reaction, ocular motility and ocular fundus. A right congruent, wedgeshaped homonymous hemianopia (fig 1) was detected. A brain CT scan disclosed an ischaemic lesion of the posterolateral area of the left thalamus, enhanced by contrast (fig 2). Eight months later the visual field was normal, and the patient had no visual complaints.

\section{Case 2}

This patient was a 49 year old male with moderate arterial hypertension, who suddenly noticed a disturbance of his left visual field with no other general or ophthalmological complaints. On neurophthalmological 
Figure 2 Brain CT scan with a left posterolateral thalamic lesions. Case 1.

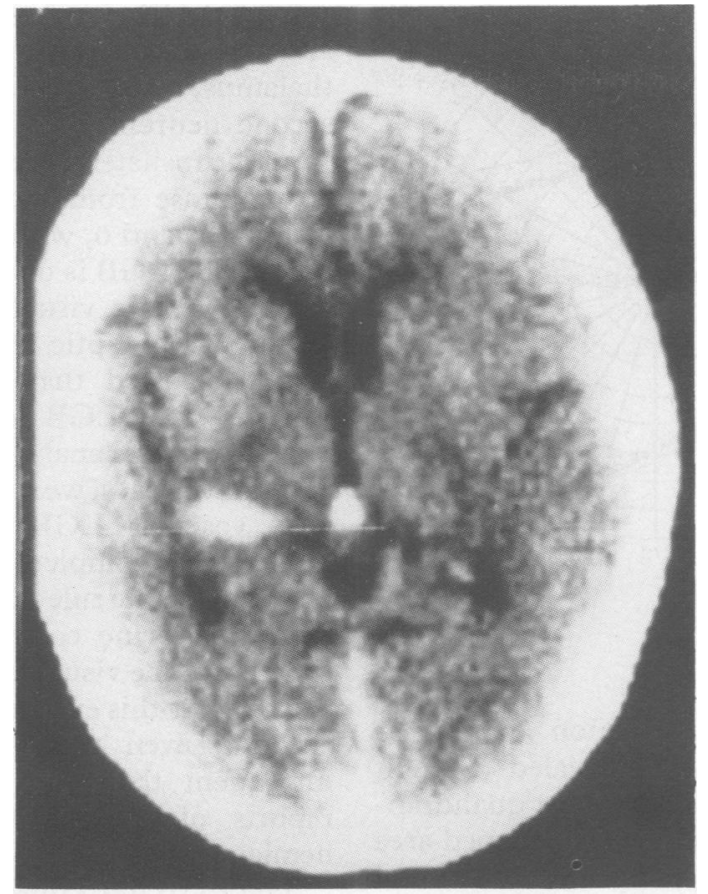

examination his visual acuity was $6 / 6$ in both eyes, the pupil reaction, ocular motility and fundus examination were normal. The visual field had an incomplete wedge-shaped left homonymous hemianopia (fig 3). The neurological examination was otherwise normal. A second generation CT scan did not disclose a definite lesion. Based on the visual field defect and a negative CT scan, an ischaemic lesion of the LGB was suggested.

\section{Case 3}

This patient was a 71 year old female with a clinical history of pulmonary tuberculosis, moderate arterial hypertension and cardiac arrythmia due to an auricular flutter. Her present illness began suddenly one night with tachycardia followed by significant polyuria and a mild left hemiparesis of sudden onset. On examination, a few hours later, the blood pressure was 150/90, with normal cardiac rhythm. On neurological examination she had a mild left sensory motor deficit. Neurophthalmological examination showed right visual acuity $6 / 18$ and left $6 / 12$, with normal pupil reactions and eye movements. The fundus examination revealed a tilted disc with atrophy of peripapillary pigment epithelium. The contrast enhanced brain CT scan showed a right thalamic and inferotemporal (hippocampus) ischaemic lesion (fig 4). The visual field disclosed a congruent left superior quadrantic defect (fig 5). Six months later the visual field had returned to normal.

\section{Case 4}

This patient was a 56 year old male with a two year history of gastrectomy for a peptic ulcer, without arterial hypertension or diabetes mellitus, who, after bending down, experienced a slight dizziness and a strange visual feeling. General and neurological examination were normal. Neurophthalmological examination showed normal visual acuity, pupillary reaction, ocular motility and fundus. On visual field examination there was a congruent left superior quadrantanopsia (fig 6). Brain CT scan showed a right posterolateral thalamic infarct (fig 7). A year later, the neurophthalmological control was normal and CT scan revealed a small hypodense lesion of the same area.

\section{Case 5}

This patient was a 56 year old woman with chronic arterial hypertension who had been treated with propranolol and diuretics. Her current illness began with a sudden onset right hemiparesis and a right visual field deficit. On general examination her blood pressure was $130 / 90$, with no other abnormality. On neurological examination there was a mild right hemiparesis and a right visual field defect on confrontation. The hemiparesis partially recovered in four hours, but the field defect showed no change. A brain CT on admission was normal. The neurophthalmological examination two days later showed normal vision in both eyes, with normal ocular

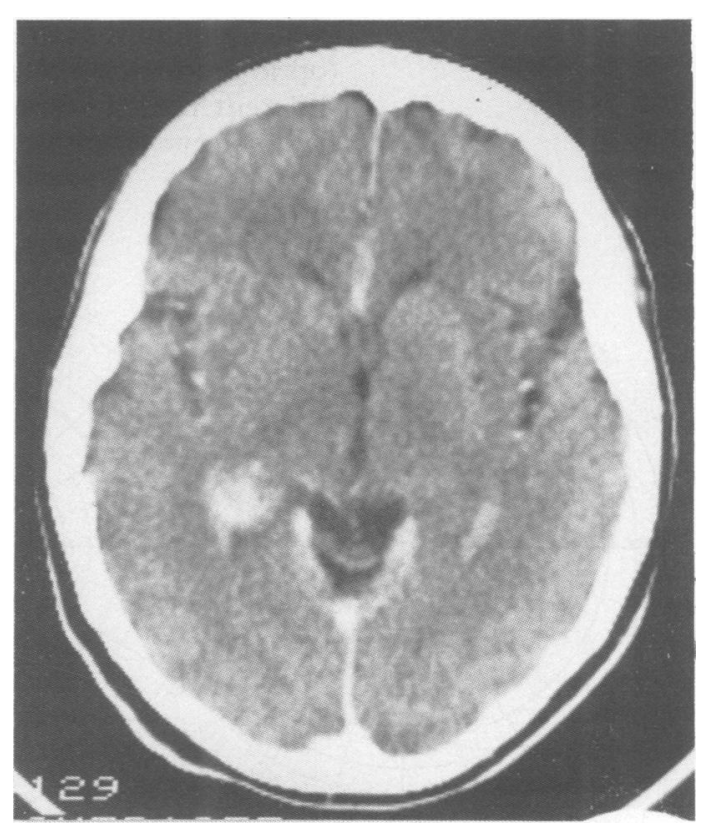

Figure 4 Brain CT scan with a right thalamic and inferotemporal ischaemic lesion. Case 3.

Figure 3 Wedge-shaped, left homonymous hemianopia. Case 2. 


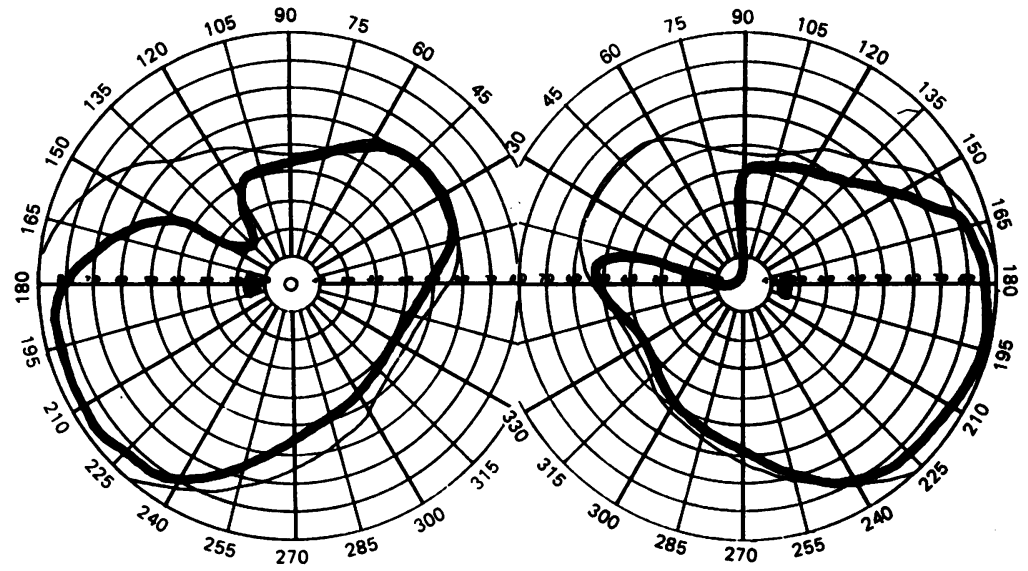

Figure 5 Left superior quadrantopsia. Case 3.

motility, pupillary light reaction and ocular fundus. Her visual field revealed a right superior and inferior homonymous quandrantic defect with sparing of a wedge-shaped area symmetrically located along the horizontal meridian (fig 8). A second CT carried out five days later showed a left basal temporal lobe ischaemic lesion (fig 9). Four vessel cerebral angiograms were normal.

\section{Discussion}

The arrangement of the nerve fibres along the visual pathway enables us to have a single brain image because retinal receptors, which receive in each eye the same external stimuli, reach a common cortical cell. The fibres of these corresponding retinal points begin their voyage 6 cms wide apart. As they proceed towards the occipital pole of the brain they approach each other and become paired as they reach the visual cortex. A discrete lesion in the posterior portion of the visual pathway has a high possibility of curtailing information coming from corresponding retinal points resulting in congruent visual field defects. The same discrete lesion in the anterior part of the pathway (optic tract) may affect fibres coming from different retinal areas producing incongruent field defects.

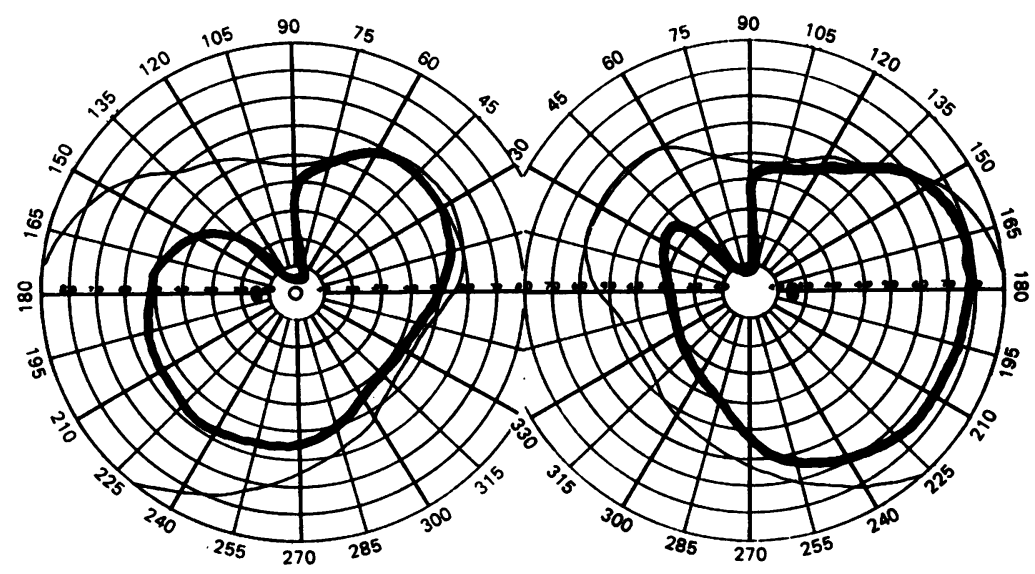

Figure 6 Left superior quadrantopsia. Case 4.
The LGB is a wedge-shaped structure situated at the posterolateral aspect of the thalamus, which receives information from the second neuron of the visual pathway. Fibres from the ipsilateral retina end in layers 2, 3 and 5 and those from the contralateral retina in layers 1,4 and 6 , without interaction between them. The LGB is on the anterior third of the retrochiasmatic visual pathway receiving the output of the optic tract. Different authors ${ }^{34}$ have suggested that visual field defects in patients with LGB lesions are incongruent. However, when analysing the literature, as well as our own cases, we conclude that field defects in a vascular LGB lesion appear to have moderate to complete congruency. This is an exception to the rule that the nearer the lesion is to the calcarine cortex the greater the congruency of the visual field defect. On analysing visual fields this exception should be taken into account, even though LGB lesions are so infrequent that they are not mentioned in reports of a large series of homonymous hemianopias. ${ }^{5}$

The LGB has a dual blood supply: from the anterior choroidal artery (branch of the internal carotid artery) and from the lateral choroidal artery (branch of the posterior cerebral artery). Frisen $e t a l^{6}$ have reported two patients with a wedge-shaped congruent homonymous hemianopia showing that the lesion was due to an occlusion of the lateral choroidal artery. The same author ${ }^{7}$ reported another patient with a superior and inferior homonymous defect which he called a quadruple sectoranopia. Cerebral angiography revealed an occlusion of the distal portion of the anterior choroidal artery and normal lateral choroidal artery. Schacklett $e t a l^{8}$ have presented an elegant scheme of the projection of the visual field on the LGB. They reported two patients with a wedge-shaped homonymous hemianopia, one with a congruent defect and another that was incongruent. The patient with the congruent

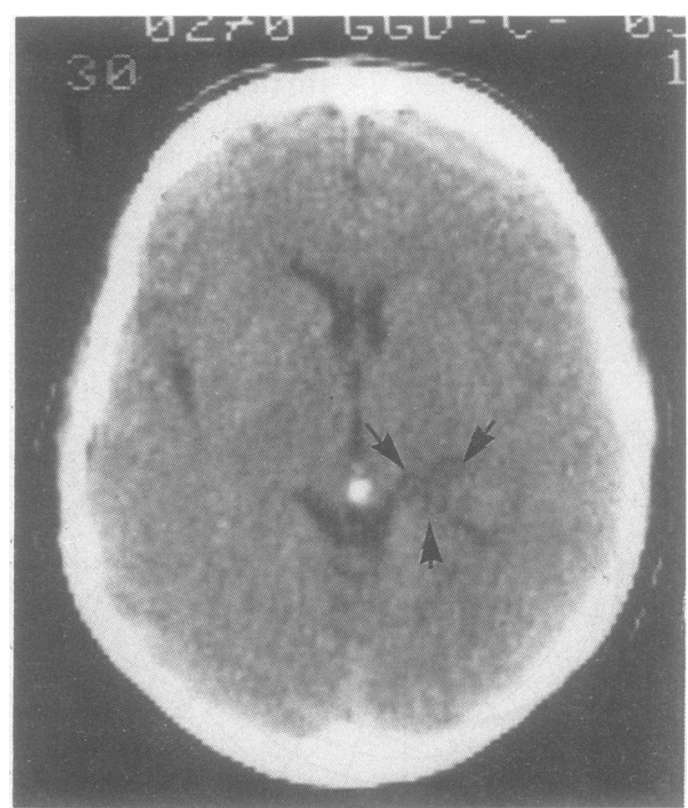

Figure 7 Brain CT scan with a right posterolateral thalamic lesion. Case 4. 


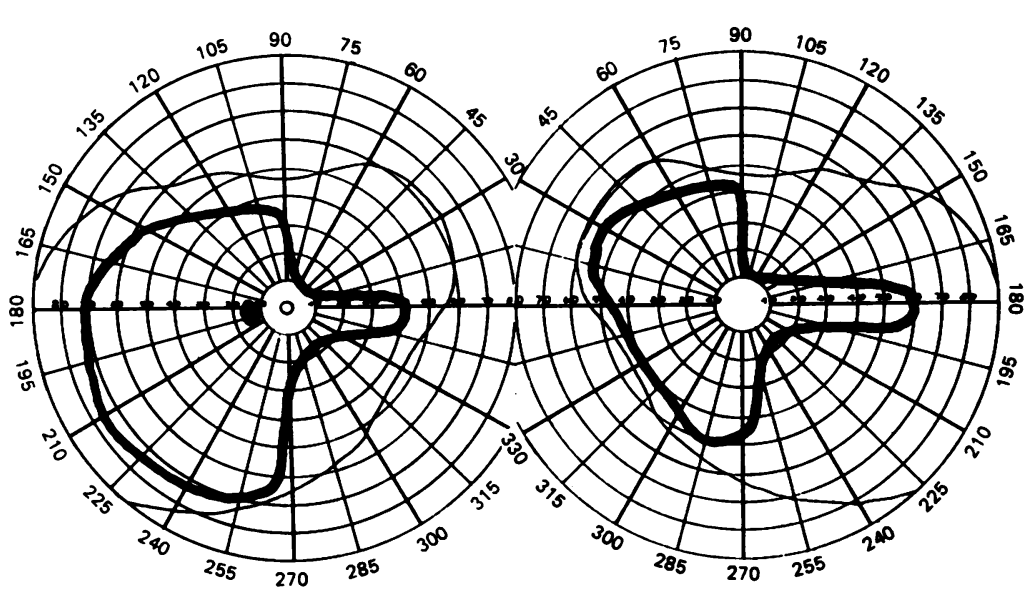

Figure 8 Right quadruple sectoranopia. Superior and inferior quadrantic defects with sparing of a central wedge-shaped area. Case 5.

defect had an ischaemic lesion of the LGB produced by an arteriovenous malformation of the lateral choroidal artery.

From this evidence we have devised a schematic diagram (fig 10) in which knowing the area of the visual field disrupted, we can conclude, within limits, which LGB arterial system is occluded-anterior choroidal or lateral choroidal artery. Two of our patients presented with typical wedge-shaped homonymous hemianopia and even though one had a negative CT scan (probably due to its low sensitivity), we believe that in both cases the same territory, that is, the area served by the lateral choroidal artery, was involved. Two other patients had a congruent superior homonymous quadrantic defect. One had an infarct of the area of the anterior choroidal artery, while the other had an ischaemic lesion of the LGB but the arterial territory involved could not be defined. The fifth patient had a superior and inferior quadrantic defect with sparing of the area just above and below the

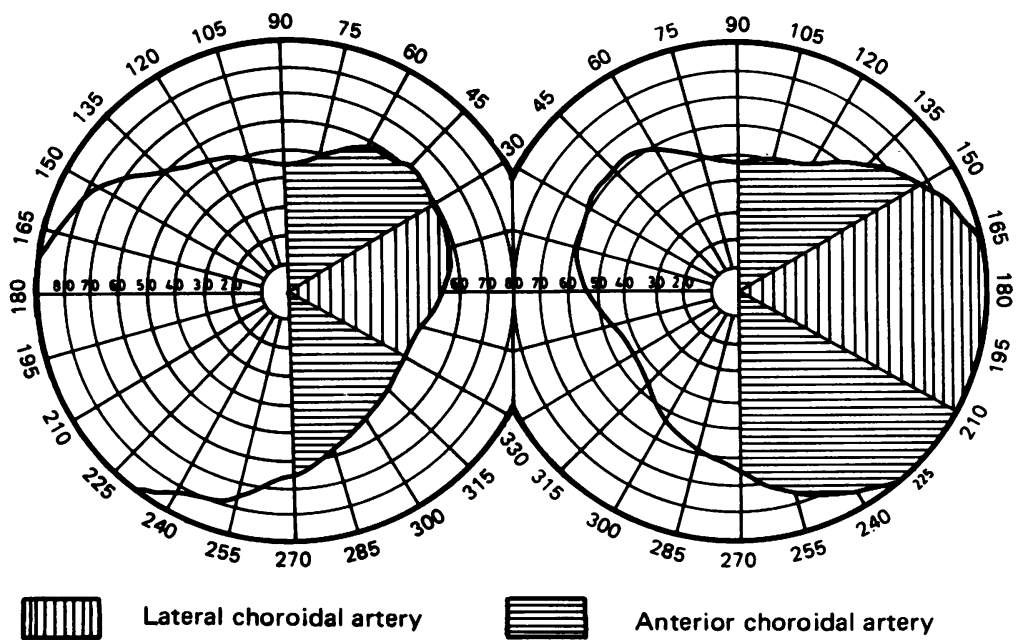

Figure 10 Area of visual field disrupted by occlusion of the anterior or lateral choroidal artery.

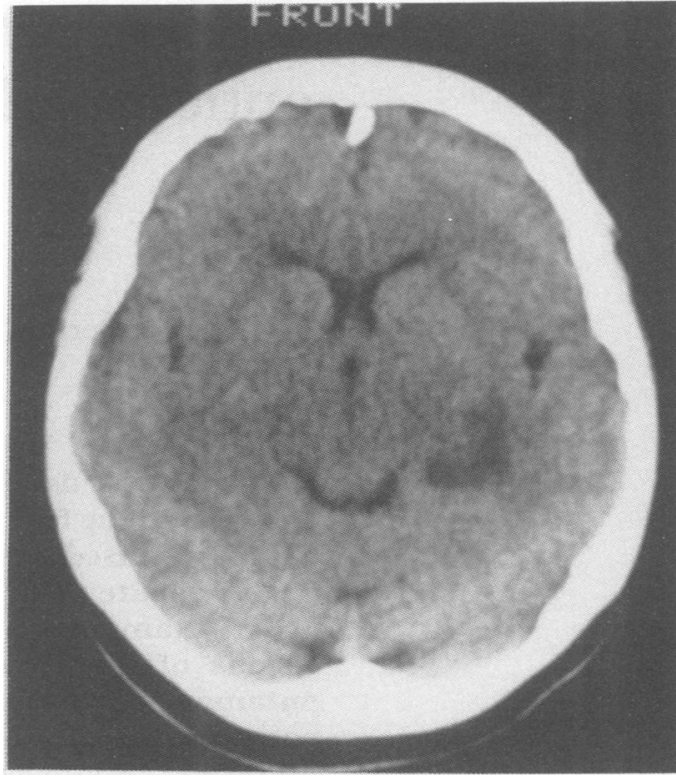

Figure 9 Brain CT scan with a left basal temporal lobe ischaemic lesion. Case 5.

horizontal meridian-the sector defect of Frisen-and the CT scan showed an ischaemic lesion of the territory supplied by the anterior choroidal artery.

In a patient with a history of a brain vascular lesion the finding of a typical wedge-shaped congruent homonymous hemianopia strongly suggests an ischaemic lesion of the LGB involving the lateral choroidal artery. Conversely, if the patient has a quadruple sectoranopia ${ }^{7}$ or a superior congruent homonymous quadrantic defect and a thalamic infarct, it is likely that there has been an ischaemic lesion involving the anterior choroidal artery. When a non cardiogenic embolic infarct is suspected, it is important to determine whether the ischaemia affects the anterior or posterior cerebral circulation as a different approach to investigation is adopted to each.

1 Harrington DO. In: The Visual Fields. 2nd edn. Saint Louis; Mosby CV. 1964:134.

2 Mohr JP, Leicester J, Stoddard T, Sidman M. Right hemianopia with memory and color deficits in circumscribed left posterior cerebral artery territory infarction. Neurology 1971;21:1104-13.

3 Geurology $1971 ; 21: 1104-13$. gruous homonymous field defect in two patients with gruous homonymous field defect in two patients with partial lesion of the lateral geniculate
Neurosurg Psychiatry 1971;34:1-6.

4 Balado M, Malbran J, Franke E. Incongruencia hemianópica derecha por lesion primitiva del cuerpo geniculado lateral izquierdo (isóptera internas). Arch Arg Neurol 1934;11:143-59.

5 Smith JL. Homonymous hemianopia: A review of one hundred cases. Am J Ophthalmol 1962;54:616-22.

6 Frisen L, Holmegaard L, Rosencrantz M. Sectorial optic atrophy and homonymous horizontal sectoranopia. A lateral choroidal artery syndrome? I Neurol Neusosurg Psychiatry 1979;42:590-4.

7 Frisen L. Quadruple sectoranopia and sectorial optic atrophy: A syndrome of the distal anterior choroidal artery. J Neurol Neurosurg Psychiatry 1979;42:590-4.

8 Shacklett D, O'Connor P, Dormant CN, Linn D, Carter J. Congruous and incongruous sectorial visual defects with lesions of the lateral geniculate nucleus. Amer J Ophthalmol 1984;98:283-90. 